revista ANTHROPOLÓGICAS

Ano 21, 28(1):188-203, 2017

\title{
'Con la Aparición de la Primera Estrella': Identidad, género y liderazgo en una comunidad judía del sur de la región chaqueña argentina
}

Armando Mudrik

Por medio de trabajo de campo propio abordamos el estudio de ideas y prácticas vinculadas al comienzo del día judío entre la comunidad de origen ashkenazi de la localidad de Moisés Ville, emplazada en el sur de la región chaqueña argentina, al norte de la provincia de Santa Fe. Particularmente partimos de las diferencias observadas entre el criterio más considerado en la tradición judía para el comienzo del día y los criterios que actualmente tiene en cuenta el grupo abordado, mostrando cómo son llevados a la práctica en el ambiente doméstico y en el plano de las ceremonias religiosas en comunidad. Al explorar estas ideas celestes logramos poner en evidencia la existencia de una flexibilidad funcional de los criterios para el comienzo del día judío y a la vez, observar vínculos con procesos de construcción de identidad, género y liderazgo.

Astronomía Cultural; Día Judío; Judíos Ashkenazim; Región Chaqueña Argentina.

\section{El origen de Moisés Ville y en la actualidad}

El presente trabajo aborda desde la óptica de la astronomía en la cultura (Ruggles \& Saunders 1993; Iwaniszewski 1990), el estudio de ideas celestes y prácticas religiosas vinculadas al comienzo del día

a Coordinador Área Astronomía y Planetario, Centro de Interpretación Científica Parque de Las Tejas. Facultad de Matemática, Astronomía y Física, Universidad Nacional de Córdoba, Argentina. Email: mudrik@oac.uncor.edu. 
judío entre la comunidad judía de Moisés Ville, localidad que se encuentra en la zona sur del departamento San Cristóbal de la provincia argentina de Santa Fe. Esta región pertenece al denominado Chaco Santafesino, al sur del Gran Chaco sudamericano.

Exploramos aqui los criterios que actualmente los miembros de la mencionada colectividad judía tienen en cuenta para considerar el inicio del día o de festividades, mostrando cómo son llevados a la práctica en el ambiente doméstico y en el plano de las ceremonias religiosas en comunidad. Al estudiar estas ideas celestes podemos observar por un lado la existencia de una flexibilidad operativa en la aplicación de los criterios para el comienzo del día judío, y por otro lado, entrever vínculos con procesos de construcción de identidad, género y liderazgo.

Esta comunicación forma parte a la vez de un amplio proyecto sobre el conocimiento de las astronomías de criollos y de los diversos colectivos de colonos inmigrantes - principalmente europeos - y sus descendientes asentados en el Chaco argentino, en el marco de un emprendimiento de diversos investigadores para abordar globalmente la astronomía de la región chaqueña (López 2015).

Realizamos la investigación a través de trabajo de campo propio en la localidad de Moisés Ville. Como la mayoría de las localidades de esta región, Moisés Ville surge durante el proceso de colonización, siendo la primera colonia agrícola judía de Argentina, establecida en 1889 (Cherjovsky 2014:30-35). En este contexto el término 'colonización' se refiere a un proceso social promovido por el estado que vinculaba el ingreso de inmigrantes europeos al país para ser ubicados en territorios chaqueños, que habían sido recientemente incorporados al estado nacional, con el fin de practicar la agricultura y ganadería. Fue un fenómeno que tuvo lugar desde mediados del siglo XIX hasta mediados de siglo XX (Gori 1988). Particularmente la idea del estado era que los inmigrantes europeos actuaran como 'fuerza civilizadora' (Gori 1988; Juliano 1987).

La comunidad judía abordada en nuestro trabajo, está originada por inmigrantes y descendientes de inmigrantes provenientes de Eu- 
ropa Central y del Este, todos pertenecientes al bloque ashkenazi (Asheri 1987:8) pero adscriptos a distintas corrientes como los ortodoxos jasidim de Ucrania, Polonia, Rumania y Rusia, y los mitnagdim de Lituania; como también los conservadores ${ }^{1}$ provenientes de Alemania y Holanda (Cherjovsky 2014:47; Asheri 1987:268-270). La principal lengua de estos grupos era el idish, con sus variantes correspondientes a cada país, las cuales aun pueden distinguirse en la actualidad entre sus descendientes. En Europa, la mayoría de los inmigrantes citados, no eran agricultores, mas bien eran profesionales, mercaderes y en algunos casos rabinos, matarifes ('shoijet') y escribas de la Torá ('soifer'). Exceptuando el conjunto fundador de Moisés Ville, familias judías fueron seleccionadas de a grupos en Europa por la Jewish Colonization Association y localizadas en terrenos pertenecientes a dicha empresa colonizadora, formando durante el período de 1891 hasta mediados de siglo XX, la colonia Moisés Ville (Cherjovsky 2014). Además de las familias 'colonizadas', se fueron sumando inmigrantes judíos espontáneos que se asentaron como obreros o comerciantes en la localidad de Moisés Ville. Muchos de los grupos de inmigrantes fuertemente nucleados en torno a su fe religiosa, traían consigo todos los elementos necesarios para desarrollar su vida litúrgica y cotidiana como lo venían haciendo hasta antes de su emigración, y una vez establecidos en la colonia, formaron asociaciones o sociedades religiosas con personería jurídica, desde las cuales se gestionaron sinagogas, cementerios y escuelas judías (Cherjovsky 2014).

Si bien también desde el inicio en la colonia y localidad de Moisés Ville residieron criollos e inmigrantes no judíos (Italianos, españoles, polacos, ucranianos, lituanos, alemanes del Volga, rusos), hasta la década de 1940 no superaban al número de pobladores judíos. Además en las últimas décadas, la población del distrito ha decrecido respecto de sus máximos históricos, pasando de unas 3500 a unas 2500 personas aproximadamente (Cherjovsky 2014:154). Debido a un recambio demográfico, la colectividad judía perdió el peso demográfico que ostentaba hasta mediados del siglo XX, y en consecuencia el creciente 
número de católicos, protestantes y testigos de Jehová que han ingresado han transformado a Moisés Ville en una sociedad heterogénea en cuanto a lo cultural, lo religioso y lo socioeconómico.

El grupo social al que pertenecen las personas entre las que realizamos el trabajo de campo representa en la actualidad una minoría, siendo aproximadamente el 10\% del total de los pobladores de Moisés Ville. Según Cherjovsky, en el año 2007, la colectividad abordada contaba con "la existencia de 164 hogares judíos que albergaban a 258 personas (incluyendo a 35 familias conformadas por matrimonios 'mixtos' o exogámicos)" (2014:155). La constante retracción demográfica que puede ser observada entre la población judía, "forma parte de un fenómeno extensible a la mayoría de las colectividades judías del interior del país" (Cherjovsky 2014:155). Este proceso ha traido aparejado el cierre de instituciones comunitarias judías y una sustancial modificación en lo que respecta a la orientación religiosa de la colectividad, observandose actualmente pocos hogares ortodoxos descendientes de los primeros inmigrantes provenientes de Europa del este, y un predominio de familias conservadoras, principalmente inmigrantes y decendientes de inmigrantes judíos alemanes de la última ola inmigratoria.

Respecto a la vida litúrgica se puede observar que solo una cuarta parte de la comunidad acude a las dos sinagogas que aún se encuentran activas durante las fechas religiosas más importantes (figura 1). Muy pocos observan los preceptos cotidianos básicos, como el descanso sabático y el consumo de alimentos 'kasher' ${ }^{3}$ o avalados por el cashrut (Asheri 1987:114). Además aproximadamente desde la década de 1970 la colectividad carece de rabino, debido a los costos que implican sus servicios comunitarios, los cuales los miembros de la comunidad no están dispuestos a solventar.

Otra de las carácterísticas que pueden observarse dentro de la colectividad israelita de Moisés Ville es el incremento de la tasa de matrimonios exogámicos, hecho que desde hace décadas trae aparejado un importante conflicto en el ceno de la dirigencia comunitaria, 
vinculado con el deseo de muchas de estas parejas de ser enterrados, ambos, en el cementerio judío.

La institución que agrupa la dirigencia comunitaria judía en Moisés Ville es la Comunidad Mutual Israelita, conocida como 'la kehila’', la cual además brinda servicios sociales, funerarios, educativos y religiosos. Las familias asociadas a la kehilá generan aportes para sostener el funcionamiento de la institución, además del cobro de los servicios funerarios y la renta que deja un campo de 300 ha donado por una socia.

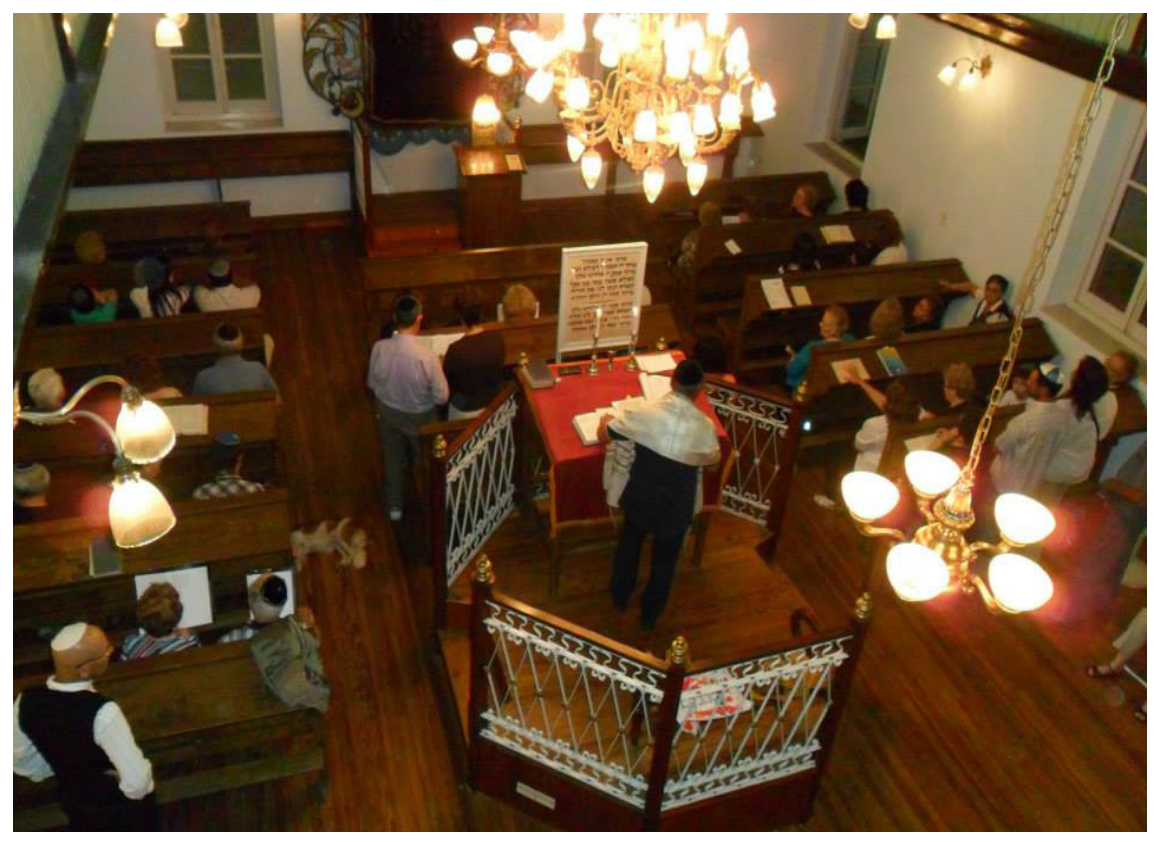

Figura 1: Oficio de Kabalat Shabat en la sinagoga 'Brener' de Moisés Ville, Santa Fe, Argentina. Noviembre de 2014. Fotografía archivo del autor.

Los anteriores y actuales líderes comunitarios han sido formados en la escuela hebrea local llamada Iahaduth ('judaísmo'), creada 
en el año 1929. La misma desde 1943 contaba con el Seminario de Maestros de Hebreo 'Yosef Draznin'. La mayoría de los entrevistados fueron estudiantes, docentes o directivos en la escuela Iahaduth y en el Seminario 'Draznin'. Durante los años noventa el Seminario tuvo que ser 'cerrado', mientras que en 2012, 'por falta de alumnos' la escuela cerró definitivamente. Aunque la colectividad actualmente podría caracterizarse etareamente por ser una comunidad compuesta por adultos y adultos mayores, existe una decena de niños y jóvenes que concurren a un espacio de educación judía no formal sustentado por aportes norteamericanos y que sigue una línea ideológica sionista.

A pesar de representar una minoría, la colectividad judía moisesvillense continúa ocupando un lugar de poder en la política local, debido a su capital patrimonial-histórico como así también a su capital económico. Los miembros de la colectividad judía, en su mayoría componen un grupo conformado por chacareros, comerciantes, profesionales y empresarios agro-ganaderos (Las actividades económicas más extendidas en la zona son la ganadería de invernada, la lechería y la cosecha de leguminosas, forrajes y cereales). Sumado a esto el sesenta por ciento de los campos localizados en la zona rural de Moisés Ville aún pertenecen a propietarios judíos cuyos aportes impositivos son importantes en el presupuesto anual del gobierno comunal.

\section{El campo etnoastronómico}

Desde que comenzamos en 2010 con nuestro trabajo de campo etnográfico entre hombres y mujeres de la colectividad judía de Moisés Ville, hemos podido observar que el conocimiento astronómico de los entrevistados en general aparece vinculado a tres aspectos importantes de su vida social: a las tareas agropecuarias, a la predicción de los fenómenos meteorológicos y al plano religioso (Mudrik 2011a, 2015a). En este sentido, si bien muchas de estas ideas ya eran consideradas en Europa por los inmigrantes que dieron origen a la comunidad abordada (Cociovitch 2005), nos resultaba interesante explorar el 
impácto del cambio de hemisferio experimentado por los inmigrantes en las prácticas e ideas religiosas (Mudrik 2011b), y también el rol del cielo en las práxis religiosas de los miembros de la colectividad judía en la actualidad. Es así que al participar con los interlocutores en ceremonias religiosas y al observar la liturgia en el ambiente doméstico, pudimos vislumbrar ideas y situaciones interesantes relacionadas con consideraciones respecto al comienzo del día judío que existen entre miembros de esta comunidad.
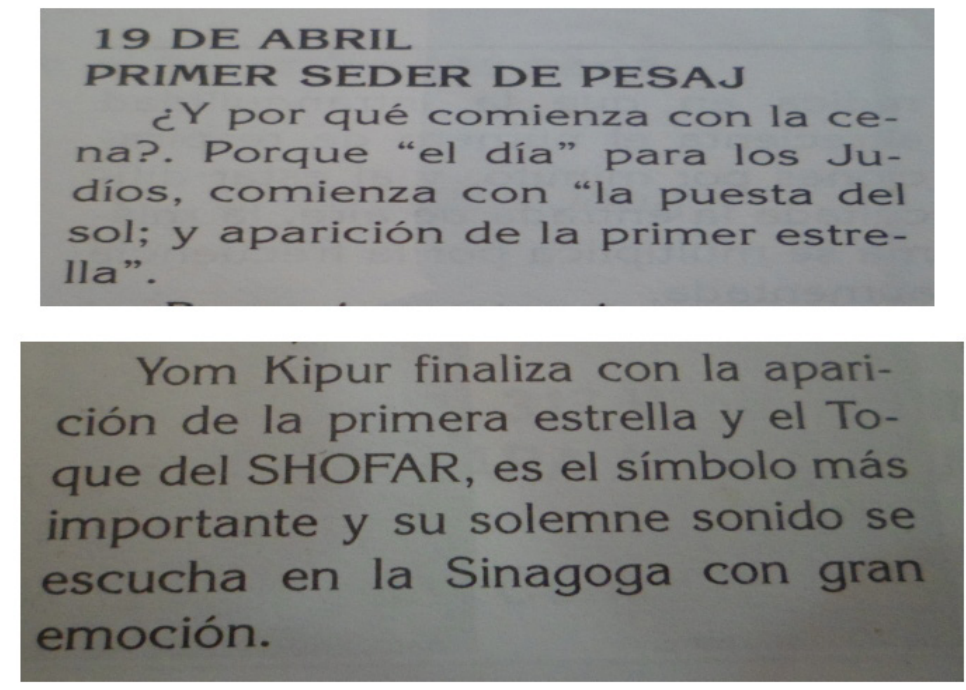

El viernes 29, con el Cabalat-Shabat (recibimiento del sábado), que se lleva a cabo con el advenimiento de la primer estrella, en el Templo Barón Hirsch, donde se congregó numeroso público, se ofició oraciones y cánticos correspondientes a este dia.

Figura 2: Extractos de notas periodísticas sobre festividades judías escritas por Lipe Notkovich en el períodico moisesvillense "Que lindo país!!! Amigos...". ARRIBA: “Pesaj' (Pascua Judía). El Nacimiento de un Pueblo”. N40, página 8, año 2000. MEDIO: "Rosh Hashana y Yom Kipur", n 57, página 15, año 2001. ABAJO: "110 años de historia. Homenaje a nuestros Padres y Abuelos. 'NO TENIENDO NADA; LO LOGRARON TODO’”, n 36, página 12, año 1999. 
Según podemos encontrar en textos que han sido mencionados como referencias importantes para esta comunidad, como el Shuljan Aruj o la obra introductoria al judaismo elaborada por Barylko (1977), quien ha sido una figura importande dentro del Seminario de Maestros de Hebreo 'Yosef Draznin'; el día judío, en particular shabat o alguna festividad, 'comienza con la puesta de sol' y termina con la 'puesta de sol' del siguiente día. Lo mismo podemos encontrar en otros textos (Feldman 1931:89; Asheri 1987; Singer 1906) siendo este criterio el más aceptado dentro de la tradición judía. Según Asheri (1987:128) y The Jewish Encyclopedia ${ }^{6}$ (Singer 1906:475), el criterio por el cual el día judío comienza con 'la puesta de sol', surge de la exégesis de uno de los vérsiculos iniciales de la Torá: Genesis 1:5, del cual se entiende que "la noche viene antes de la mañana, en el computo del día” (Asheri 1987:128).

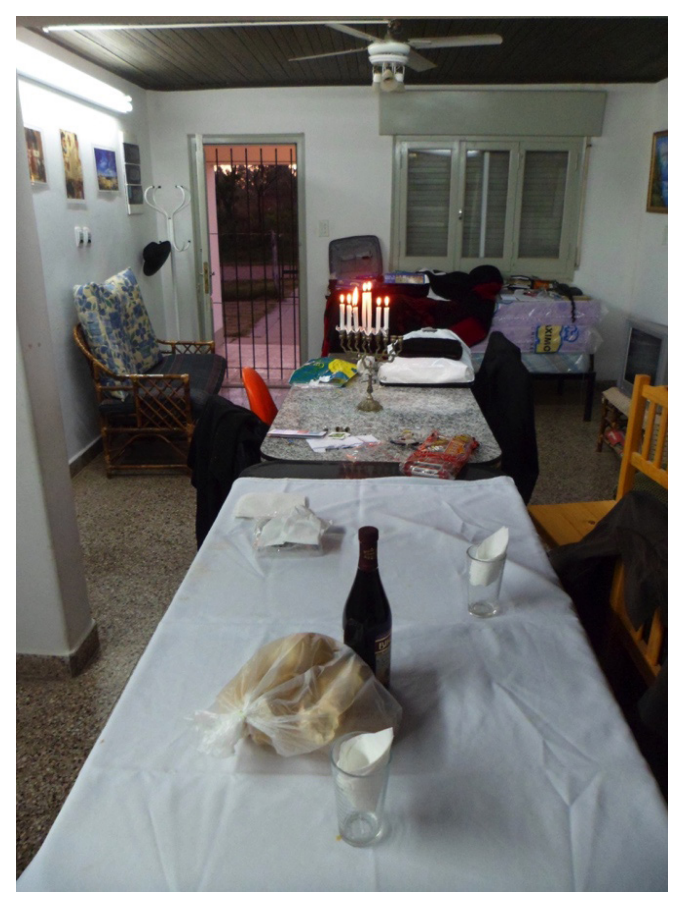

Figura 3: Momento del encendido de velas antes del Shabat en un hogar judío en Moisés Ville. Mayo de 2013. Fotografía archivo del autor. 


\section{Distintas ideas vinculadas al día}

Ahora bien este criterio que considera 'la puesta de sol' como evento astronómico para definir el inicio de shabat o cualquier otro día judío, difiere un poco de los que hemos registrado en campo. Participando de varios Kabalat Shabat u oficios para 'recibir el Shabat' (sábado), el día 'sagrado' o 'más importante' de la semana, en los templos Barón Hirsch y Brener de Moisés Ville, hemos podido recoger entre varios de los interlocutores que "el día comienza" o "Shabat comienza cuando sale la primera estrella" o "con la aparición de la primera estrella”, haciendo referencia claramente al momento en que luego de la puesta del sol puede observarse la primera estrella en el firmamento. Podemos además encontrar referencias a este criterio en notas periodísticas sobre festividades judías celebradas en Moisés Ville en publicaciones locales escritas por miembros de la comunidad judía local (ver figura 2). Es interesante señalar que esta idea sobre el momento en el que comienza el día para los judíos fue relevada entre miembros de la comunidad adscriptos como 'conservadores' o 'tradicionalistas', etre ellos muchas maestras hebreas egresadas del Seminario de Maestros de Hebreo 'Yosef Draznin', que a la vez integran la comisión de 'la Kehilá, y que para la mayoría de nuestros interlocutores son consideradas 'las que más saben’ sobre tradición judía en Moisés Ville.

Aunque el criterio del comienzo del día 'con la aparición de la primera estrella' es el más escuchado, dos miembros de la comunidad adscriptos como ortodoxos afirman que Shabat o 'el día sagrado' comienza y termina "con la puesta de sol y la observación de por lo menos tres estrellas", criterio que uno de los entrevistados considera con origen 'en la Torá'.

Resulta importante aquí mencionar que cuando comentabamos este criterio de 'las tres estrellas' entre algunas de las maestras de hebreo miembro de 'la Kehilá', ellas afirmaban "que no era cierto" y que lo mejor era que le "pregunte a algún rabino conservador".

De modo accesorio, sólo para mostrar la diversidad de ideas celestes que nos han señalado algunos interlocutores considerados 'con- 
servadores', 'tradicionalistas' o 'poco religiosos', mencionamos que existen ideas como por un lado la que hace referencia a que 'el lucero es la estrella de los rezos' , y por otro, la que la observación de ciertas 'estrellas' marcan el comienzo de determinadas 'fiestas'.

Por último, sólo encontramos una referencia explícita a 'la puesta de sol', vinculando este fenómeno con el comienzo de Shabat entre algunos interlocutores durante una de las plegarias del oficio de $\mathrm{Ka}$ balat Shabat, denominada lejá dodí. En esta oración el jazán (cantante ritual que dirije el oficio) pide que "en la estrofa numero diez" todos de pie miren hacia "la entrada" del templo. En este caso, para la mayoría de los informantes que aprendieron y conocen "el significado" de la plegaria, "cuando se dice la última estrofa, se gira para recibir a la novia que es el sábado" , 'shabat amalká' y que "entre por la puerta, simbólicamente"; pero algunos de los feligreses de la sinagoga Barón Hirsch, en ese momento de la oración piensan que "se mira a la puesta del sol", "despidiendo al todo poderoso", siendo importante mencionar que en este templo el ingreso principal se encuentra orientado 'al oeste'.

\section{De las ideas a la práctica}

Ante la diversidad de ideas relacionadas a eventos celestes que marcan el comienzo del día judío recogidas entre los distintos interlocutores, cabe preguntarse ¿Cómo son considerados en la práctica estos criterios? Según lo que hemos observado, en la práctica nadie mira el cielo meticulosamente con el fin de registrar el momento preciso en el que 'aparece la primera estrella' o 'las tres primeras estrellas', y así 'recibir' shabat o alguna festividad, sino que más bien en algunos casos sólo observan 'la claridad del cielo' y la mayoría utiliza un 'luaj' (calendario hebreo), para el año judío correspondiente. Un 'luaj' es un almanaque que contiene las fechas del calendario hebreo correlacionadas con el correspondiente calendario gregoriano. En el mismo se encuentran registradas las fechas de inicio de festividades, días sa- 
grados como el shabat, días de ayuno y demás fechas importantes en el calendario liturgico judío. Además en un 'luaj' podemos encontrar tanto la hora de inicio del día o festividad (puesta del sol), como la hora indicada para el encendido de velas momento antes del inicio del shabat (ver figura 3), tarea que tradicionalmente es asignada a las mujeres (Asheri 1987:129). Claro está que estos horarios están calculados para la localidad en la que se confecciona el 'luaj'.

Entre nuestros entrevistados hemos notado que utilizan almanaques elaborados por comunidades judías de la ciudades de Buenos Aires, Tucumán y Córdoba, que en algunos casos son enviados como obsequios a la comunidad judía de Mosés Ville o en otros son comprados por los miembros de la colectividad. Estos almanaques son colgados en lugares visibles en los hogares y algunas mujeres los 'llevan' en sus 'carteras'. Aunque la mayoría de nuestras interlocutoras utilizan los horarios fijados en un 'luaj' para conocer el momento de encender las velas antes del shabat, muchas de ellas también prestan atención a 'la claridad del cielo' para concretar esta tarea, sin cumplir estrictamente con los horarios indicados por la tradición. Es interesante que para algunos entrevistados, entre ellos uno ortodoxo, consideran que shabat comienza primero para las mujeres, registrandose afirmaciones tales como: "a las mujeres les llega primero el Shabat". Esta seguramente es una interpretación surgida debido a que como dijimos anteriormente, la tarea de encendido de velas es tradicionalmente asignada a mujeres.

Por otro lado, en el plano de las actividades religiosas comunitarias, hemos observado que particularmente 'la kehilá' utiliza desde hace décadas para cáda año el correspondiente 'luaj' que la Asociación Mutual Israelita Argentina (AMIA) elabora con horarios para la ciudad de Buenos Aires. Según registramos, este 'luaj' solo contiene los horarios de inicio de días como shabat y otras festividades y son estos horarios los que son tenidos en cuenta por la comisión de 'la kehilä' de Moisés Ville para fijar en particular durante todo el año el horario de comienzo de los oficios de Kabalat Shabat en las sinagogas de la lo- 
calidad. Si bien el inicio de los oficios se dan próximos a los horarios establecidos en el 'luaj', las ceremonias se fijan con un horario que rige durante varios meses pero que "va variando a lo largo del año" teniendo en cuenta los horarios indicados en el 'luaj' y buscando un compromiso "para que la mayoría [de los miembros de la colectividad] pueda asistir"

Es interesante que si bien muchos de los entrevistados que no pertenecen a la comisión de 'la kehilà' conocen que la misma es la que decide los horarios de comienzo de los oficios, muy pocos saben cómo se deciden precisamente a estos horarios, desconociendo que 'la kehilá' utiliza un 'luaj' con horarios de comienzo días judíos para la ciudad de Buenos Aires. En este sentido, la mayoría considera que para fijar los horarios de los oficios en Moisés Ville, 'mas o menos se tiene en cuenta cuando se pone el sol'.

Para finalizar, es oportuno mencionar que dado que en determinados períodos del año los oficios comienzan en horarios en los que, según consideraciones realizadas por la minoría ortodoxa, 'ya comenzó shabat', esta flexibilidad con la cual 'la kehilá' decide el comienzo de los oficios religiosos, es entendida dentro de un conjunto más amplio de decisiones tomadas por la mencionada institución, como acciones que apuntan a la 'pérdida de identidad' y 'decadencia' de la comunidad judía de Moisés Ville.

\section{Palabras finales}

El presente trabajo intenta mostrar cómo a partir de trabajo de campo dirigido a explorar ideas celestes entre miembros de un grupo social y analizando sus resultados dentro del marco de la astronomía cultural o en este caso de la etnoastronomía (Lopez 2016), logramos observar procesos de construcción de identidad, género y liderazgo desarrollados en la sociedad abordada.

Particularmente, primero hemos puesto en evidencia cómo una sociedad entiende y utiliza el conocimiento astronómico, reinterpretando y elaborando concepciones o ideas que luego son llevadas a la 
práctica de una manera conveniente a los intereses del grupo. En este sentido, hemos registrado que para la comunidad judía abordada, en la observación del cielo a la hora de fijar criterios para la determinacion del comienzo de festividad o shabat, la precisión no es algo que se persigue ya que se busca una flexibilidad que resulte operativa.

Por otro lado también vimos cómo los distintos criterios para fijar los eventos astronómicos que determinan el inicio del día judío son considerados por los miembros de la colectividad como un espacio de disputa de saberes y por lo tanto también de poder o liderazgo, pero también nos muestran que esas diferencias de criterios hacen a la identidad de los grupos dentro de la comunidad judía en cuestión.

Además hemos visto el importante rol que actualmente desempeña un grupo de lideres mujeres en la comunidad judía de Moisés Ville, no solo como referentes del conocimiento de la tradición judía sino también como agentes con capacidad de dirigir una comunidad ante los desafíos que le plantea el problema de la constante reducción demógrafica.

\section{Notas}

1 En este trabajo, utilizamos el adjetivo 'conservador' para todo aquello adscripto a la corriente judaica del conservadorismo, movimiento que surge en Alemania a mediados de siglo XIX. Sus diferencias con la ortodoxia tradicional son básicamente en el plano ritual. Por ejemplo: mujeres y hombres pueden rezar juntos sin físicas separaciones, parte de los rezos pueden decirse en el idioma nacional, la cabeza ha de estar cubierta en los oficios religiosos pero no necesariamente en otras ocasiones (Barylko 1977).

2 Término propio del castellano regional de Santa Fe, que hace referencia al proceso de asentar un grupo de personas en tierras para practicar agricultura y ganadería. Surge a partir del gran proceso de colonización durante fines de siglo XIX y principios de siglo XX en la provincia de Santa Fe y es hasta hoy utilizado por descendientes de colonos inmigrantes.

3 La expresión kasher, propia de la tradición judía sefaradí, equivale a kosher y significa puro o apropiado (Asheri, 1987). Quizás los entrevistados utilizan el término kasher dado que los maestros de las 'escuelas hebreas' para la formación religiosa 
presentes en las colonias judías a principios de siglo XX, eran provenientes de Francia y de origen sefaradí.

4 'La Kehilâ', comunidad en hebreo, es una organización con personería jurídica que dirige y financia la vida comunitaria judía en Moisés Ville. Es la encargada de organizar las ceremonias y festividades judías, a la vez que financia el mantenimiento del cementerio, las sinagogas, la escuela hebrea entre otras instituciones judías y apoya toda acción constructiva a favor del Estado de Israel. Surgió a partir de la Jevra Kedusha (sociedad de entierros) en 1891 y en 1923 obtuvo personería jurídica. Dede 1969, por una disposición nacional atinente a las asociaciones étnicas, pasó a denominarse Comunidad Mutual Israelita de Moisés Ville. Los miembros o autoridades de 'la Kehila' son elegidos "por elecciones libres y democráticas" por voto de los socios con cuota al día mayores de veintidós años que tengan una antigüedad mayor a un año. El mandato dura dos años y los cargos incluyen a un presidente, dos vicepresidentes, secretario, tesorero y doce vocales. Actualmente los cargos son ocupados principalmente por mujeres y sus decisiones son tomadas mediante reuniones entre los integrantes de la organización. Fuente: Estatutos de la Comunidad Mutual Israelita de Moisés Ville, 1970.

5 Nombre con el que se conoce a la obra donde se sistematiza y organiza todo el material existente sobre leyes y preceptos que reglamentan la vida judía en todos sus aspectos. Ampliamente aceptado y consultado con sus anexos en el mundo ashkenazí, fue redactado en el siglo XVI por el rabino Iosef Caro (Barylko 1977:192).

6 Todos los volúmenes de la edición en ruso de la Jewish Encyclopedia editada entre 1906 y 1913 en San Petesburgo, se encuentran en la Biblioteca de la Sociedad Kadima, ahora dependiente de la Comunidad Mutual Israelita de Moisés Ville.

7 'El lucero' es un asterismo u objeto brillante en el cielo señalado por algunos interlocutores como "la estrella más brillante del cielo" la cual en un determinado contexto es observada antes del amanecer o como en nuestro caso después de la puesta del sol, siendo "la estrella que aparece primero".

8 Los horarios fijados para el inicio de los oficios de Kabalat Shabat para el año 2016 fueron: Enero: 20hs; Febrero: 20hs; Marzo hasta la mitad de Abril: 19:30; Segunda mitad de Abril has la primera mitad de Septiembre: 19hs; Segunda mitad de Septiembre hasta Noviembre: 19:30; Diciembre: 19:45.

\section{Referencias}

ASHERI, Michael. 1987. O Judaismo Vivo: As tradições e as Leis dos Judeos Praticantes. Rio de Janeiro: Imago.

BARYLKO, Jaime. 1977. Introducción al Judaísmo. Buenos Aires: Fleishman \& Fischbein Editores.

CHERJOVSKY, Iván. 2014. De la Rusia Zarista a la Pampa Argentina: Memoria e identidad en las colonias de la Jewish Colonization Association. Tesis de doctorado. Buenos Aires: Universidad de Buenos Aires. 
COCIOVITCH, Noe. 2005. Génesis de Moisés Ville. Buenos Aires: Milá.

FELDMAN, W. M. 1931. Rabbinical Mathematics and Astronomy. New York: Hermon Press.

GORI, Gastón. 1988. Inmigración y Colonización en la Argentina. Buenos Aires: EU. DEBA.

JULIANO, Dolores. 1987. "El discreto encanto de la adscripción étnica voluntaria”. In RINGUELET, Roberto (ed.): Procesos de Contacto Interétnico, pp. 83 112. Buenos Aires: Ediciones Búsqueda.

IWANISZEWSKI, Stanisław. 1991. "Astronomy as a Cultural System". Interdisciplinarni Izsledvaniya, 18:282-288.

LÓPEZ, Alejandro Martín. 2015. "Astronomy in the Chaco Region, Argentina". In RUGGLES C. (ed.): Handbook of Archaeoastronomy and Ethnoastronomy, Pp. 987- 995. New York: Springer.

2016. Los cielos del chaco. Pp 145- 201 en: GIMÉNEZ BENITEZ, Sixto y GOMEZ, Cecilia (eds.). Primera Escuela Interamericana de Astronomía Cultural. Universidad Nacional de La Plata. Facultad de Ciencias Astrónomicas y Geofísicas. Observatorio Astronómico de La Plata: La Plata.

MUDRIK, Armando. 2011a. "A eucalyptus in the moon: folk astronomy among European colonists in northern Santa Fe province, Argentina". In RUGGLES, Clive (Ed.): Archaeoastronomy and Ethnoastronomy: Building bridges between cultures, pp. 84-92. Cambridge: Cambridge University Press.

. 2011b. "Interacción cultura-ambiente de los primeros colonos inmigrantes judíos de la prov. de Santa Fe". Actas del X Congreso Argentino de Antropología Social. La antropología interpelada: nuevas configuraciones político-culturales en América latina. Buenos Aires: Editorial de la Facultad de Filosofía y Letras, Universidad de Buenos Aires.

. 2015. "Ethnoastronomy in the Multicultural Context of the Agricultural Colonies in Northern Santa Fe Province, Argentina”. In RUGGLES, Clive (ed.): Handbook of Archaeoastronomy and Ethnoastronomy, pp. 997-1004. New York: Springer.

RUGGLES, C. \& SAUNDERS, N. 1993. "The Study of Cultural Astronomy". In RUGGLES, C. \& SAUNDERS, N. (eds.): Astronomies and Cultures, pp. 1-31. Niwot: University Press of Colorado.

SINGER, Isidore. 1906. Jewish Encyclopedia. Vol. 4. New York: Funk and Wagnalls

Resumo: Por meio de trabalho de campo próprio, abordamos o estudo de ideias e práticas vinculadas ao começo do dia judaico entre a comunidade de origem ashkenazi da localidade de Moisés Ville, no sul da região do Chaco argentino, no norte da província de Santa Fé. Particularmente partimos das diferenças observadas entre o critério mais considerado na tradição judaica para o começo do dia e os critérios que atualmente são utilizados pelo grupo abordado, mostrando como são postos em prática no ambiente do- 
méstico e nas cerimônias religiosas na comunidade. Ao explorar estas ideias celestes, conseguimos evidênciar a existência de uma flexibilidade funcional dos critérios para o início do dia judaico e, ao mesmo tempo, observar os vínculos com os processos de construção de identidade, gênero e liderança.

Palavras-chave: Astronomia Cultural; Dia Judaico; Judeus Ashkenazita; Chaco Argentino.

Abstract: We study ideas and practices related to the beginning of the Jewish day through ethnographic field research among the Ashkenazi community of the town of Moisés Ville, located in the southern Argentinean Chaco, in northern area of the province of Santa Fe. Particularly, we carried out a survey of the most considered criterion for the beginning of the Jewish day that the Jewish community currently takes into account, showing how they are put into practice in the domestic environment and in the plane of Religious ceremonies in community. Exploring these celestial ideas, we have demonstrated the existence of a functional flexibility of the criteria for the beginning of the Jewish day and at the same time to observe links with processes of construction of identity, gender and leadership.

Keywords: Cultural Astronomy, Jewish Day, Ashkenazi Jews, Argentinean Chaco, Ethnoaestronomy.

Recebido em fevereiro de 2017.

Aprovado em junho de 2017. 